\title{
Mathematical Models of Tumor Growth and Angiogenesis
}

\author{
M. Can \\ International University of Sarajevo
}

\begin{abstract}
.
In this paper we present several mathematical models of tumor growth and angiogenesis expressed by systems of ODE's encoding the most essential observations and assumptions about the complex hierarchical interactive processes of tumor neo-vascularization (angiogenesis). The simplest modeling option presented merely captures the three independent variables mentioned earlier-tumor size $\mathrm{N}$, total vessel volume $\mathrm{V}$ and the amount of protein $\mathrm{P}$. We modify this model assuming that the protein is additionally consumed by growing vessels and obtain a model with protein consumption. Next models with time-delays are introduced. To make our models more realistic, two more compartments representing more complex vascularity and protein effects are introduced. Hence five dimensional models are obtained.
\end{abstract}

1991 Mathematics Subject Classification. 34C23, 34G20, 34K14, 34K18, 34K60, 37G10, 37N25, 92C30, 92C50.

Key words and phrases. Three dimensional angiogenesis, five dimensional angiogenesis, time-delay differential equations, angiogenesis.

\section{INTRODUCTION}

While there had been substantial achievements in the field, there has been made so little progress in the last three decades of war against cancer. In 1980's there was a consensus in the biomedical research community that cancer progresses hazardously and, hence, does not yield to prediction at any level of accuracy. In general, it was believed that biological systems are too complex to be accurately retrieved by mathematical models. This declarable mistrust in the power of the biomathematics left the biomedical sciences lagging behind other sciences, as an immature sequel of experimental observations.

During the same years bio mathematicians constructed among the others the resonance theory of population persistence in randomly fluctuating environments, which was later applied to cancer chemotherapy.

Macroscopic models are another set of efforts to the same direction. They assume logistic tumor growth do not take into account vessel maturation and don't allow for the nutrient dependency secretion of proangiogenic factors. 
Models describing vascular tumor system on three scales: molecules, cells, and macroscopic entities are not complete since it does not include maturation of new blood vessels.

Other continuous models define three major processes together: tumor growth, growth factor production and vessel growth by order of complexity.

Growth of malignant tumors beyond the diameter of $1-2 \mathrm{~mm}$ critically depends on their neo-vascularization, which provides vital nutrients and growth factors, and also clears toxic waste products of cellular metabolism [12]. Indeed, the role of angiogenesis - the formation of new blood vessels by budding from existing ones - as a target for cancer therapy, is currently a focus of intensive research [12],[8],[19].

In order to establish successful antiangiogenic treatment rationale, the dynamics of angiogenesis must be better understood. These dynamics are very complex, involving many interacting oscillatory processes, which operate on several scales of time and space. Their essential constituents are briefly described below.

Having reached a certain size and, therefore, a certain critical volume/surface ratio, a shortage of oxygen (denoted hypoxia) and nutrients is created within the tumor. Under hypoxia the tumor produces proteins, notably Vascular Endothelial Growth Factor (V EGF). Increasing V EGF levels lead to increased proliferation and mobility of endothelial cells, and, as a result, to increased formation of immature vessels by these cells. Consequently, the blood supply of the tumor is augmented, encouraging tumor proliferation [22],[11],[25],[23].

The protein V EGF also ensures integrity of immature vessels, and its insufficiency leads to their regression
[5],[18],[17]. Immature vessels undergo a process of maturation by attaching a layer of pericytes. Vessel maturation is enhanced by the binding of the protein Angiopoietin-1 (Ang1), which is secreted by tumor cells, to Tie 2 receptors on endothelial cells [10],[24]. Both the immature vessels and the tumor produce the protein Angiopoietin-2 (Ang2), which blocks the Ang1-Tie2 bond, thus stopping the maturation process and inducing the reverse process - the destabilization of the mature vessels [21],[20],[1]. It appears, then, that Ang1/Ang2 ratio determines which of the processes - maturation or destabilization is the dominating one [2].

The present talk is devoted to the investigation of a simpler family of angiogenesis models which still captures the essential properties of the system. We introduce multi-parameter families time-delay ODE systems, which encode the basic known facts and assumptions concerning tumor induced angiogenesis processes. We believe that for these models it makes most sense to ask qualitative questions involving behavior of the system locally with respect to time.

\section{GENERAL ASSUMPTIONS OF THE MODELS.}

Each of the models herein described involves the following time dependent variables [1]:

- the number of tumor cells or tumor size (denoted by $\mathrm{N}$ ),

- the concentrations of growth factors known to be involved in tumor angiogenesis, defined as P. For a more accurate description, $\mathrm{P}$ may be broken down into several growth factors (proteins) which may differ in effects and/or kinetics,

- the volume of blood vessels feeding the tumor which, again, may either be defined separately as the volumes of 
immature and mature vessels, or as the total of both, denoted by V .

All modeling alternatives are systems of ordinary differential equations with or without time-delay. In all of these models we use "sigmoid like" functions smooth monotonous functions having a horizontal asymptote, e.g. $1 /\left(1+e^{k(x+s)}\right)$. These functions describe a response of the system to the relevant biological stimuli. The reason for such a choice of the response function is the experimental observation that, below and above certain thresholds, changes in the intensity of the stimuli have minor effects on the response. Between the threshold values (in the sensitivity region), the process rate monotonously depends on the stimuli value. In our analysis we use only the basic properties of sigmoidal functions and we do not expect their exact shape to be easily determined from experiments or otherwise.

We assume that the tumor size dynamics are determined by availability of oxygen and nutrients. The amount of nutrients delivered to the tumor is proportional to the volume of blood vessels feeding the tumor, whether inside the tumor or in its close vicinity. To take this into account we use vessel density which may relate to immature vessels, mature vessels, or the total of both, denoted by E1, E2 or E,

respectively. The vessel density is calculated by dividing the corresponding vessel volume by the tumor size $\mathrm{E}=\mathrm{V} / \mathrm{N}$ . To simplify our models we assume that vessel wall permeability (perfusion) is the same for immature and mature vessels. For the tumor size dynamics in all our models we assume the Malthusian law determined by

$$
\dot{N}=f_{1}(E) N(t)
$$

where $f_{1}$ is an increasing sigmoid function capturing the processes of cell proliferation and death:

$$
\left.f_{1}(E)\right|_{E=0}<0, \lim _{x \rightarrow \infty} f_{1}(x)>0
$$

For dynamics of protein (growth factor) compartments we assume that proteins are produced by tumor cells or immature vessels, and degraded by an intrinsic clearance process. Elaboration of the clearance process will be discussed later, suggesting the introduction of additional consuming elements, such as the forming vessels, into the model.

We assume that the dynamics of vessel compartments are a superposition of four processes, some are contrasting some of the others: formation of immature vessels, regression of immature vessels, maturation of immature vessels and destabilization of mature vessels into immature vessels. We assume that these four processes are driven by sigmoid like responses, as described above, depending on specified proteins. These proteins are the stimuli mentioned earlier as the effectors of these functions.

\section{A THREE-DIMENSIONAL MODEL WITH NO TIME- DELAY.}

The simplest modeling option presented merely captures the three independent variables mentioned earlier-tumor size $\mathrm{N}$, total vessel volume $\mathrm{V}$ and the amount of protein $\mathrm{P}$. The only thing that we assume about the protein is that it drives the vessel formation or regression in a sigmoidal way. The rate of change of $\mathrm{N}$ is determined by a Malthusian law sigmoidally depending on $\mathrm{E}$ (representing EVD, as defined earlier). The protein $\mathrm{P}$ is produced by the tumor at a rate sigmoidally dependent on $\mathrm{E}$ and is decaying at a constant positive 
rate $\delta$. The rate of change of the vessel volume $\mathrm{V}$ is also sigmoidally driven by the protein. Thus we have the system [1]

$$
\left\{\begin{array}{l}
\dot{N}=f_{1}(E) N \\
\dot{P}=f_{2}(E) N-\delta P \\
\dot{V}=f_{3}(P) V
\end{array}\right.
$$

where

- $f_{1}$ is the tumor cells proliferation rate; it is an increasing function of $E$ and satisfies (2).

- $f_{2}$ is the protein production rate; it is a decreasing function of $\mathrm{E}$ and satisfies

$f_{2}(x)>0, \quad \lim _{x \rightarrow \infty} f_{2}(x)=0$

- $f_{3}$ is the vessel growth rate; it is an increasing function of $P$ and satisfies

$$
\left.f_{3}(P)\right|_{P=0}<0, \lim _{x \rightarrow \infty} f_{3}(x)>0
$$

To simplify the analysis we make a substitution of variables $V=E . N$ and get the system

$$
\left\{\begin{array}{l}
\dot{N}=f_{1}(E) N \\
\dot{P}=f_{2}(E) N-\delta P \\
\dot{E}=f_{3}(P) E-f_{1}(E) E
\end{array}\right.
$$

\section{EXAMPLES}

As the functions satisfying conditions (2), (4) and (5)

$$
\begin{aligned}
& f_{1}(E)=\frac{d_{1} E^{2}}{1+E^{2}}-a_{1}, f_{2}(E)= \\
& \frac{a_{2}}{1+E}, f_{3}(P)=\frac{d_{3} P^{2}}{1+P^{2}}-a_{3}
\end{aligned}
$$

For the parameter set $\left\{a_{1}, a_{2}, a_{3}, d_{1}, d_{3}, \delta\right\}=\{1 ., 1 ., 0.5,2 ., 1 ., 1$. the system (6) has three equilibrium points:

$$
\begin{aligned}
& \text { parmval }=\left\{\left\{a_{1}, a_{2}, a_{3}, d_{1}, d_{3}, \delta\right\}\right. \\
& \text { parmval1 }=\{1 ., 1 ., 0.5,2 ., 1 ., 1 .\}
\end{aligned}
$$

Trivial critical point $C O=\{0,0,0\}$

Eigenvalues at $C O: E O=\{-1,-1, .5\}$ unstable saddle

Smitrivial critical point

$C 1=\{0,0,1 / \sqrt{3}\}$

Eigenvalues at $C 1: E 1=\{-1,-.75,-.5\}$ stable node

Nontrivial critical point $C 2=\{2,1,1\}$

Eigenvalues at $C 2$ : $E 2=\{-1.13+.76 \mathrm{I}$,1.13-.76I,.27\} unstable saddle

Dynamics of the system is sensitive to the initial conditions near unstable saddle points. Integration of the system for two initial conditions are shown belaow:

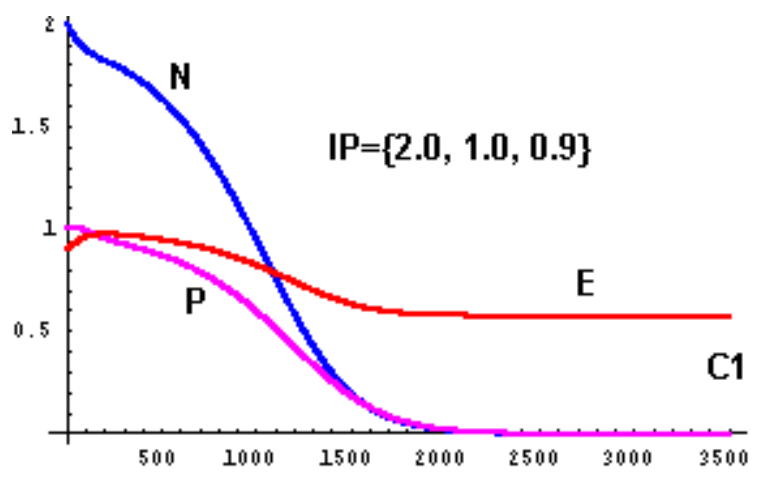

Figure 1. For the initial point $\{2 ., 1 ., .9\}$ system stabilizes at the stable node $C 1=\{0,0,1 / \sqrt{3}\}$

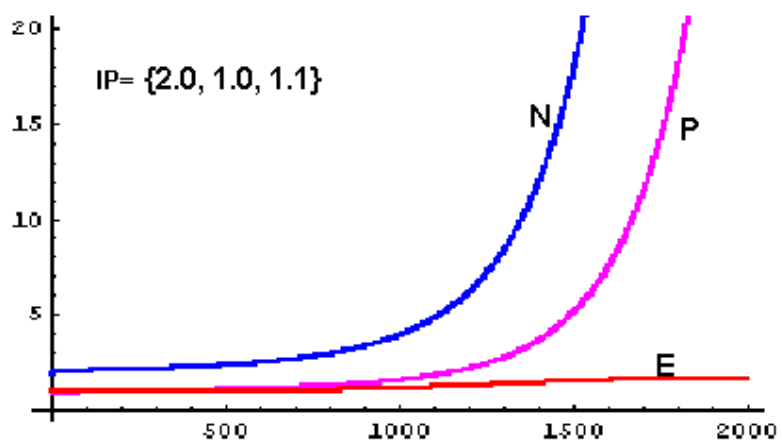

Figure 2. For the initial point $\{2 ., 1 ., 1.1\}$ $\mathrm{P}(\mathrm{t})$ get stabilized at, while $\mathrm{N}(\mathrm{t})$ and $\mathrm{E}(\mathrm{t})$ diverged towards large values.

For another parameter set $\left\{a_{1}, a_{2}, a_{3}, d_{1}, d_{3}, \delta\right\}=\{.5 ., 1 ., 1 ., 1 ., 1 ., 2\}$ the system (6) has two equilibrium points: 
Trivial critical point $C O=\{0,0,0\}$

Eigenvalues at $C O: E O=\{-1,-.5,-.5\}$ stable node

Nontrivial critical point $C 1=\{2,1,1\}$

Eigenvalues at $C 1: E 1=\{-.91+.87 \mathrm{I}$,.91-.87I,.32\} unstable saddle

Dynamics of the system is sensitive to the initial conditions near unstable saddle points. Integration of the system for two initial conditions $\{1,1,1\}$ are shown below:

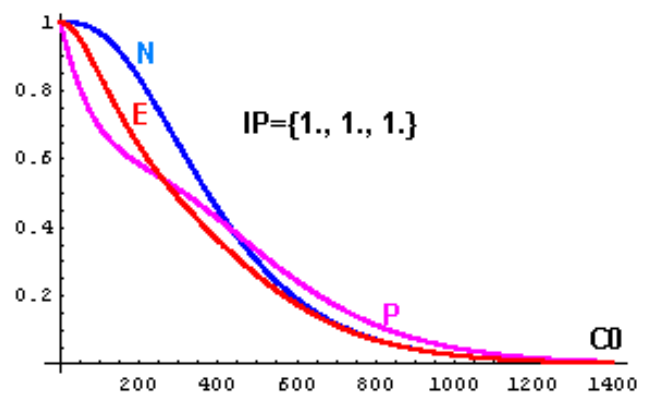

Figure 3. For the initial point $\{1 ., 1 ., 1$. system stabilizes at the stable node $C O=\{0,0,0\}$

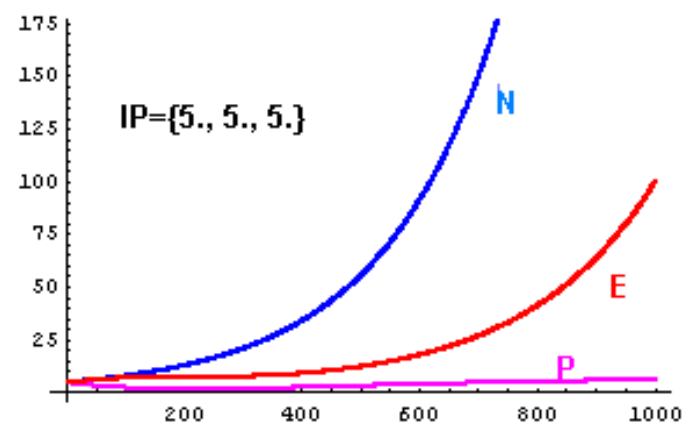

Figure 4. For the initial point $\{5 ., 5 ., 5$. $\mathrm{P}(\mathrm{t})$ stabilized at , while $\mathrm{N}(\mathrm{t})$ and $\mathrm{E}(\mathrm{t})$ diverged towards large values.

\section{A MODEL WITH PROTEIN CONSUMPTION.}

We can modify the model assuming that the protein is additionally consumed by growing vessels. Thus, for the dynamics of the protein compartment we modify the second equation in (3) [1]

$$
\left\{\begin{array}{l}
\dot{N}=f_{1}(E) N \\
\dot{P}=f_{2}(E) N-\delta P-f_{c}\left(f_{3}(P) E N\right) P \\
\dot{E}=f_{3}(P) E-f_{1}(E) E
\end{array}\right.
$$

where

$$
f_{c}(x)>0, \quad \lim _{x \rightarrow \infty} f_{c}(x)>0, \quad f_{c}(0) \approx 0(9)
$$

\section{MODELS WITH TIME-DELAYS.}

Two time-delays are introduced in (3): $\tau_{1}$ in the proliferation/death response to stimuli and $\tau_{2}$ in the vessel formation/regression response to stimuli [1].

Let $E_{\tau_{1}}=E\left(t-\tau_{1}\right), \quad P_{\tau_{2}}=P\left(t-\tau_{2}\right)$, then system (3) is modified, yielding:

$\left\{\begin{array}{l}\dot{N}=f_{1}\left(E_{\tau_{1}}\right) N \\ \dot{P}=f_{2}(E) N-\delta P \\ \dot{E}=\left(f_{3}\left(P_{\tau_{2}}\right)-f_{1}\left(E_{\tau_{1}}\right)\right) E\end{array}\right.$

EXAMPLE: The system (10) is integrated with the parameter values parmval 1 and time delays $\tau_{1}=\tau_{2}=100$ :

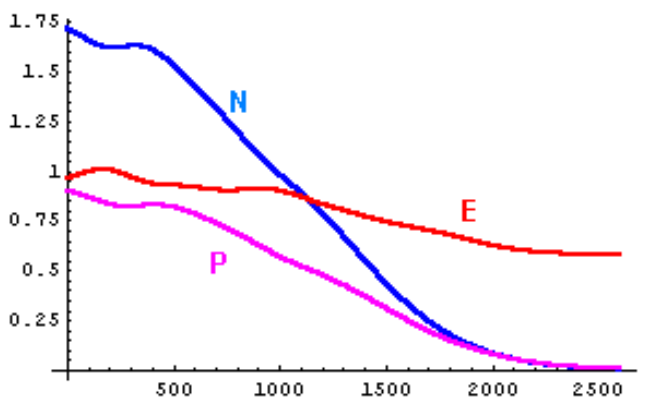

Figure 5. For the initial point $\{2 ., 1 ., .9\}$ system stabilizes at the stable node $C 1=\{0,0,1 / \sqrt{3}\}$

6. FIVE DIMENSIONAL MODELS. 
To make our models more elaborate and realistic, we introduce more compartments representing more complex vascularity and protein effects [1]. First, the inclusive representation of vessels volume $\mathrm{V}$ is replaced by separate descriptions of the immature and mature vessel volumes denoted by $V_{1}$ and $V_{2}$, respectively. The values of either vessel subpopulation will be separately analyzed. Hence, the model allows both maturation of immature vessels and destabilization of mature vessels. Secondly, the general term protein, denoted $P$, is now replaced by two specific proteins namely V EGF, denoted $P_{1}$ and Ang1, denoted $P_{2}$. We assume that V EGF is produced by the tumor at a rate sigmoidally dependent on the vessel density and decays at a constant rate $\delta_{1}$, and that Ang1 is produced by the tumor at a constant rate $\alpha$ and decays at a constant rate $\delta_{2}$.

Note that another growth factor, Ang2, is not modeled here as an additional dimension. Rather, it is assumed to exist in a constant amount. Hence, it is represented as one of the constant parameters wherever relevant in the functions $f_{1}, f_{2}, f_{3}$. Let us also introduce the characteristic time-delays as follows: $\tau_{1}$ for tumor proliferation and death, $\tau_{2}$ for immature vessel formation and regression, and $\tau_{3}$ for mature vessel destabilization. We get the system:

$$
\left\{\begin{array}{l}
\dot{N}=f_{1}\left(E_{\tau_{1}}\right) N \\
\dot{P}_{1}=f_{2}(E) N-\delta_{1} P_{1} \\
\dot{P}_{2}=\alpha N-\delta_{2} P_{2} \\
\dot{V}_{1}=f_{3}\left(P_{1 \tau_{2}}\right) V_{1}-f_{4}\left(P_{2}\right) V_{1}+f_{5}\left(P_{2 \tau_{3}}\right) V_{2} \\
\dot{V}_{2}=f_{4}\left(P_{2}\right) V_{1}-f_{5}\left(P_{2 \tau_{3}}\right) V_{2}
\end{array}\right.
$$

where satisfy $(2,4,5)$ and

- $f_{4}$ is the maturation rate, it is a positive increasing function of $P_{2}$.
- $f_{5}$ is the destabilization rate, it is a positive decreasing function of $P_{2}$ and satisfies

$$
\lim _{x \rightarrow \infty} f_{5}(x)=0
$$

After making the substitutions

$$
\begin{aligned}
& V_{1} \rightarrow E_{1}=\frac{V_{1}}{N}, \quad V_{2} \rightarrow E_{2}=\frac{V_{2}}{N}, \\
& E_{2} \rightarrow E=E_{1}+E_{2}
\end{aligned}
$$

we get the system

$$
\left\{\begin{array}{l}
\dot{N}=f_{1}\left(E_{\tau_{1}}\right) N \\
\dot{P}_{1}=f_{2}(E) N-\delta_{1} P_{1} \\
\dot{P}_{2}=\alpha N-\delta_{2} P_{2} \\
\dot{E}=f_{3}\left(P_{1 \tau_{2}}\right) E_{1}-f_{1}\left(E_{\tau_{1}}\right) E \\
\dot{E}_{1}=f_{3}\left(P_{1 \tau_{2}}\right) E_{1}-f_{4}\left(P_{2}\right) E_{1}+ \\
f_{5}\left(P_{2 \tau_{3}}\right)\left(E-E_{1}\right)-f_{1}\left(E_{\tau_{1}}\right) E_{1}
\end{array}\right.
$$

Introduced above were several modeling suggestions describing angiogenesis, with or without time-delays. As demonstrated in examples, whenever time-delay was introduced into the tumor proliferation or the neovascularization process, oscillatory behavior is observed. In contrast, a timedelay in the mature vessels destabilization process does not seem to be necessary for the existence

of oscillations in the tumorvolume/vessel-density system. This might mean that an appropriate candidate for describing the system in question is the alternative that includes time-delays in the tumor proliferation or angiogenesis process.

Moreover, recall that for rationalizing the empirical results it was necessary to introduce a significant time-delay between the tumor and vessels processes. This might underline the significance of time-delays in tumor growth dynamics. It seems to us worthwhile to examine these results in the clinical context, that is, to check 
whether or not one can control tumor growth by imposing (by use of certain drugs) time-delays in the processes neovascularization.

\section{REFERENCES}

[1] Z.Agur, L.Arakelyan, P. Daugulis, Y. Ginosar, Hopf point analysis for angiogenesis models, Discrete and Cont. Dyn. Sys.-Series B 4(1)(2004), 29-38.

[2] S.A.Ahmad, et al, Differential expression of Angiopoietin-2 in colon carcinoma, Cancer

92(2001), 1138-43.

[3] L.Arakelyan, V.Vainstein, Z.Agur, Optimizing anti-angiogenic therapy using mathematical

tools, Proceedings of American Society of Clinical Oncology (ASCO) 21(2002), 440a. 1.

[4] L.Arakelyan, Y.Merbl, Z.Agur, Vessel-density balance determines tumour growth: Computer

model validated in murine-implanted human ovarian carcinoma, submitted.

[5] L.E.Benjamin, E.Keshet, Conditional switching of vascular endothelial growth factor

(V EGF) expression in tumors: Induction of endothelial cell shedding and regression of

hemangioblastoma-like vessels by V EGF withdrawal, PNAS 94(1997), 87618766.

$38 \quad$ Z.AGUR, L.ARAKELYAN, P.DAUGULIS, Y.GINOSAR

[6] L.E.Benjamin, et al, A plasticity window for blood vessel remodeling is defined by pericyte

coverage of the preformed endothelial network and is regulated by PDGF-B and V EGF,

Development 125(9)(1998), 1591-8.

[7] L.E.Benjamin, et al, Selective ablation of immature blood vessels in established human tumors

follows vascular endothelial growth factor withdrawal, J. Clin. Invest. 103(2)(1999),

$159-65$
[8] P.Carmeliet and R.K.Jain, Angiogenesis in cancer and other diseases, Nature 407(2000), 249-257.

[9] C. Chicone, "Ordinary Differential Equations with Applications", Springer Verlag, 1998.

[10] S.Davis, T.H.Aldrich, P.F.Jones, A.Acheson, D.L.Compton, V.Jain, T.E.Ryan, J.Bruno,

C.Radziejewski, $\quad$ P.C.Maisonpierre, G.D.Yancopoulos, Isolation of Angiopoietin-1, a Ligand

for the Tie-2 receptor, by Secretion-Trap Expression Cloning, Cell 87(1996), 11611169.

[11] Y.Dor, R.Porat, E.Keshet, Vascular endothelial growth factor and vascular adjustments to

perturbations in oxygen homeostasis, AJP Cell Physiology 280(6)(2001), C1367-C1374.

[12] J.Folkman, Angiogenesis in cancer, vascular, rheumatoid and other disease, Nat.Med.

1(1)(1995), 27-31.

[13] A.Gilead, M.Neeman, Dynamic remodelling of the vascular bed precedes tumor growth:MLS

ovarion carcinoma spheroids implanted in nude mice, Neoplasia 1(1999), 226230.

[14] V.Goede, T.Schmidt, S.Kimmina, D.Kozian, H.G.Augustin, Analysis of blood vessel maturation

processes during cyclic ovarion angiogenesis, Lab Invest 78 (11)(1998), 1385-94.

[15] J.K.Hale and S.M.Verduyn Lunel, "Introduction to Functional Differential Equations", volume

99 of Applied Mathematical Sciences, Springer Verlag, 1993.

[16] A.J.Hayes, et al., Expression and function of angiopoietin-1 in breast cancer, Br. J. Cancer 83(2000), 1154-60.

[17] S.J.Holash, G.D.Wiegandand, G.D.Yancopoulos, New model of tumor angiogenesis: dynamic 
balance between vessel regression and growth mediated by angiopoietin and $\mathrm{V}$ EGF, Oncogene 18(1999), 5356-5362.

[18] R.K.Jain, N.Safabakhsh, A.Sckell, Y.Chen, P.Jiang, L.E.Benjamin, F.Yuan, E.Keshet,

Endothelial cell death, angiogenesis, and microvascular function after castration in an

androgen-dependent tumor: Role of vascular endothelial growth factor, Proc.Nat. Acad.

Science USA 95.18(1998), 10820-10825.

[19] R.S.Kerbel, Tumor angiogenesis: past, present and the near future, Carcinogenesis

21.3(2000), 505-515.

[20] K.Koga, et al, Expression of Angiopoietin-2 in human glioma cells and its role for angiogenesis,

Cancer Res. 61(2001), 6248-54.

[21] P.C.Maisonpierre, C.Suri, P.F.Jones, S.Bartunkova, S.J.Wiegand, C.Radziejewski,

D.Compton, J.McClain, T.H.Aldrich, N.Papadopoulos, T.J.Daly, S.Davis, T.N.Sato,

G.D.Yancopoulos, Angiopoietin-2, a Natural Antagonist for Tie2 Tha Disrupts in vivo Angiogenesis,

Science 277:5322(1997), 55-60.
[22] A.Namiki, E.Brogi, M.Kearney, E.A.Kim, T.Wu, T.Couffinhal, L.Varticovski, J.M.Isner,

Hypoxia Induces Vascular Endothelial Growth factor in Cultured Human Endothelial Cells, JBC 270 (52)(1995), 31189-31195.

[23] H.Oh, H.Takagi, K.Suzuma, A.Otani, M.Matsumura, Y.Honda, Hypoxia and vascular endothelial growth factor selectively up-regulate Angiopoietin-2 in bovine microvascular endothelial

cells, J.Biol.Chem. $274 \quad$ (22)(1999), 15732-15739.

[24] C.Suri, P.E.Jones, S.Patan, S.Bartunkova, P.C.Maisonpierre, S.Davis, T.S.Sato,

G.D.Yancopoulos, Requisite role of Angiopoietin-1, a Ligand for the TIE2 Receptor, during

Embryonic Angiogenesis, Cell 87(1996), 1171-1180.

[25] H.T.Yuan, S.P.Yang, A.S.Woolf, Hypoxia up-regulates angiopoietin-2, a Tie-2 ligand, in

mouse mesangial cells, Kidney International 58 (5)(2000), 1912-1919.

E-mail address: mcan@ius,edu.ba 